PROCEEDINGS OF THE

AMERICAN MATHEMATICAL SOCIETY

Volume 133, Number 5, Pages 1325-1333

S 0002-9939(04)07787-1

Article electronically published on November 19, 2004

\title{
A NEW DEGREE BOUND FOR INVARIANT RINGS
}

\author{
JIANJUN CHUAI
}

(Communicated by Bernd Ulrich)

\begin{abstract}
In this paper we give a new degree bound for polynomial invariant rings of finite groups and give some applications.
\end{abstract}

\section{INTRODUCTION}

Let $\mathbf{F}$ be a field of characteristic $p \geq 0, V$ an $n$-dimensional vector space over $\mathbf{F}$, and $\mathbf{F}[V]$ the symmetric algebra of the dual, $V^{*}$, of $V$. Thus, if we choose a basis, $\left\{x_{1}, \ldots, x_{n}\right\}$, for $V^{*}, \mathbf{F}[V]$ can be identified with the polynomial algebra $\mathbf{F}\left[x_{1}, \ldots, x_{n}\right]$. Take a finite subgroup $G \leq G L(V)$. The action of $G$ on $V$ induces an action on $V^{*}$ given by

$$
g \cdot \ell:=\ell \circ g^{-1}, \forall g \in G, \ell \in V^{*} .
$$

This action further extends to an action by degree-preserving algebra automorphisms on $\mathbf{F}[V]$. The invariant ring of $G$ is the subring of $\mathbf{F}[V]$ defined by

$$
\mathbf{F}[V]^{G}:=\{f \in \mathbf{F}[V] \mid g \cdot f=f, \forall g \in G\} .
$$

It is well known that $\mathbf{F}[V]^{G}$ is always finitely generated as a graded algebra.

We say that $G$ is modular if $p$ divides the order of $G$. Otherwise, $G$ is called non-modular.

For any graded Noetherian $\mathbf{F}$-algebra $R$ of Krull dimension $d$, there are $d$ homogeneous elements $f_{1}, \ldots, f_{d}$ (called a homogeneous system of parameters) such that $R$ is a finitely generated module over $\mathbf{F}\left[f_{1}, \ldots, f_{d}\right]$ (see [5, Corollary 2.4.8]). If $R$ is free over $\mathbf{F}\left[f_{1}, \ldots, f_{d}\right], R$ is called Cohen-Macaulay. The Noether number of $R$, denoted $\beta(R)$, is the minimal integer $m \geq 0$ such that $R$ can be generated as an algebra by homogeneous elements of degree at most $m$. The embedding dimension of $R$, denoted $\operatorname{edim}(R)$, is the number of the elements in a minimal homogeneous generating set of $R$. By the graded Nakayama Lemma (see [5, Lemma 3.5.1]), $\operatorname{edim}(R)$ is well-defined.

It was proved by Noether in [13] that if $p=0$ or $p>|G|$, then $\beta\left(\mathbf{F}[V]^{G}\right) \leq|G|$. For a general non-modular group $G$, the question whether $\beta\left(\mathbf{F}[V]^{G}\right) \leq|G|$ holds (called the "Noether Gap") remained open for quite a while. Only recently this problem was solved independently by Fleischmann and Fogarty (see [8] and [9]). However, not much is known about the upper bounds of $\beta\left(\mathbf{F}[V]^{G}\right)$ when $G$ is

Received by the editors September 23, 2003 and, in revised form, January 30, 2004.

2000 Mathematics Subject Classification. Primary 13A50.

Key words and phrases. Invariant ring, degree bound.

This research was partially supported by NSERC. 
a modular group. This paper gives a general degree bound for $\beta\left(\mathbf{F}[V]^{G}\right)$ from which a new degree bound for $p$-permutation representations is derived. A result of Fleischmann and a result of Goebel follow easily as corollaries.

\section{THE THEOREM}

In the following we denote $\mathbf{F}[V]$ by $R$. For any subgroup $H \leq G$ the relative transfer $\operatorname{Tr}_{H}^{G}$ is defined by

$$
\begin{aligned}
\operatorname{Tr}_{H}^{G}: R^{H} & \longrightarrow R^{G}, \\
f & \longrightarrow \sum_{g \in G / H} g(f),
\end{aligned}
$$

where $G / H$ denotes a set of representatives of the left cosets of $H$ in $G$. It is clear that $\operatorname{Tr}_{H}^{G}$ is a well-defined homomorphism of $R^{G}$-modules. Let $R=\mathbf{F}\left[a_{1,1}, \ldots, a_{1, n_{1}}\right]$ with $a_{1 i}$ homogeneous and for any non-trivial subgroup $H \leq G$, let $\left\{a_{H, 1}, \ldots, a_{H, n_{H}}\right\}$ $\subseteq R^{H}$ be homogeneous such that their images in $R^{H} / \sum_{Q<H} \operatorname{Tr}_{Q}^{H}\left(R^{Q}\right)$ generate $R^{H} / \sum_{Q<H} \operatorname{Tr}_{Q}^{H}\left(R^{Q}\right)$ as an $\mathbf{F}$-algebra. So we have

$$
R^{H}=\mathbf{F}\left[a_{H, 1}, \ldots, a_{H, n_{H}}\right]+\sum_{Q<H} \operatorname{Tr}_{Q}^{H}\left(R^{Q}\right) .
$$

We now have the following result.

Lemma 2.1. In the above settings,

$$
R^{G}=\sum_{H \leq G} \operatorname{Tr}_{H}^{G}\left(\mathbf{F}\left[a_{H, 1}, \ldots, a_{H, n_{H}}\right]\right) .
$$

Proof. We use induction on $|G|$. If $|G|=1$, then the lemma is trivially true by assumption. So we assume $|G|>1$. Let $G_{1}, \ldots, G_{t}$ be all the maximal subgroups of $G$. For any subgroup $H \leq G_{i}$, we have

$$
\operatorname{Tr}_{H}^{G}\left(R^{H}\right)=\operatorname{Tr}_{G_{i}}^{G}\left(\operatorname{Tr}_{H}^{G_{i}}\left(R^{H}\right)\right) \subseteq \operatorname{Tr}_{G_{i}}^{G}\left(R^{G_{i}}\right) .
$$

So

$$
R^{G}=\mathbf{F}\left[a_{G, 1}, \ldots, a_{G, n_{G}}\right]+\sum_{i=1}^{t} \operatorname{Tr}_{G_{i}}^{G}\left(R^{G_{i}}\right)
$$

By induction,

$$
R^{G_{i}}=\sum_{H \leq G_{i}} \operatorname{Tr}_{H}^{G_{i}}\left(\mathbf{F}\left[a_{H, 1}, \ldots, a_{H, n_{H}}\right]\right)
$$

Thus

$$
\operatorname{Tr}_{G_{i}}^{G}\left(R^{G_{i}}\right)=\sum_{H \leq G_{i}} \operatorname{Tr}_{H}^{G}\left(\mathbf{F}\left[a_{H, 1}, \ldots, a_{H, n_{H}}\right]\right),
$$

and the result follows.

We now consider a special case. Assume $H \leq G$ is a non-trivial subgroup such that $H$ permutes the basis $\left\{x_{1}, \ldots, x_{n}\right\}$ of $V^{*}$. For any subgroup $Q \leq H$, let $N_{Q, 1}, \ldots, N_{Q, n_{Q}}$ denote the orbit products in the $Q$-set $\left\{x_{1}, \ldots, x_{n}\right\}$. It is well known that $R^{Q}$ is spanned over $\mathbf{F}$ by the orbit sums of the monomials in the $x_{i}$ 's, and a monomial is fixed by $Q$ if and only if it can be written as a monomial in the $N_{Q, i}$ 's. Furthermore, any such orbit sum is the image of a monomial in the orbit under the relative transfer $\operatorname{Tr}_{K}^{Q}$ for some proper subgroup $K<Q$ if the length of 
the orbit is greater than 1 (clearly $K$ here can be taken to be the stabilizer of the monomial under consideration). So, we have

$$
R^{Q}=\mathbf{F}\left[N_{Q, 1}, \ldots, N_{Q, n_{Q}}\right]+\sum_{K<Q} \operatorname{Tr}_{K}^{Q}\left(R^{K}\right)
$$

(here, as usual, $\sum_{K<Q} \operatorname{Tr}_{K}^{Q}\left(R^{K}\right)=0$ if $Q=1$ ).

By Lemma 2.1 we obtain

Corollary 2.2. Let $H$ be a subgroup of $G$ and assume that $H$ permutes the basis $\left\{x_{1}, \ldots, x_{n}\right\}$ of $V^{*}$. Then

$$
R^{H}=\sum_{Q \leq H} \operatorname{Tr}_{Q}^{H}\left(\mathbf{F}\left[N_{Q, 1}, \ldots, N_{Q, n_{Q}}\right]\right) .
$$

Now let us go back to the general setting. For any subgroup $H \leq G$, let

$$
\beta_{H}:=\beta\left(R^{H} / \sum_{Q<H} \operatorname{Tr}_{Q}^{H}\left(R^{Q}\right)\right),
$$

and let

$$
n_{H}:=\operatorname{edim}\left(R^{H} / \sum_{Q<H} \operatorname{Tr}_{Q}^{H}\left(R^{Q}\right)\right)
$$

So we can write

$$
R^{H}=\mathbf{F}\left[a_{H, 1}, \ldots, a_{H, n_{H}}\right]+\sum_{Q<H} \operatorname{Tr}_{Q}^{H}\left(R^{Q}\right)
$$

with $a_{H, i}$ homogeneous and $\operatorname{deg}\left(a_{H, i}\right) \leq \beta_{H}$. Furthermore, if $H \neq 1$, define

$$
\begin{gathered}
e(H):=\max \left\{n_{Q} \mid 1<Q \leq H\right\}, \\
\beta(H):=\max \left\{\beta_{Q} \cdot(|G: Q|-1) \mid 1<Q \leq H\right\},
\end{gathered}
$$

and

$$
\bar{\beta}(H):=\max \left\{\beta_{Q} \cdot|G: Q| \mid 1<Q \leq H\right\} .
$$

If $H=1$, define all the $e(H), \beta(H)$ and $\bar{\beta}(H)$ to be zero.

We have

Theorem 2.3. Let $H$ be a subgroup of $G$ such that $|G: H|$ is invertible in $\mathbf{F}$. Let $\left\{f_{1}, \ldots, f_{n}\right\}$ be a homogeneous system of parameters for $R^{G}$. Then

$$
\beta\left(R^{G}\right) \leq \max \left\{e(H) \cdot \beta(H), \bar{\beta}(H), \mathrm{d}(f),\left(\sum_{i=1}^{n} \operatorname{deg}\left(f_{i}\right)\right)-n\right\},
$$

where $\mathrm{d}(f)=\max \left\{\operatorname{deg}\left(f_{i}\right) \mid 1 \leq i \leq n\right\}$.

Proof. If $H=1$, then $R^{G}$ is Cohen-Macaulay (see 6], or [5, Theorem 3.4.1]). By Broer's result (see [1], or [5. Theorem 3.9.8]), $R^{G}$, as a module over $\mathbf{F}\left[f_{1}, \ldots, f_{n}\right]$, is generated by invariants of degree at most

$$
\left(\sum_{i=1}^{n} \operatorname{deg}\left(f_{i}\right)\right)-n
$$


Since the $f_{i}$ 's together with the module generators generate $R^{G}$ as an algebra, the theorem is true in this case. Now suppose $|H|>1$. Since $R$ as an $\mathbf{F}\left[f_{1}, \ldots, f_{n}\right]$ module is generated by polynomials of degree at most

$$
\left(\sum_{i=1}^{n} \operatorname{deg}\left(f_{i}\right)\right)-n
$$

$\operatorname{Tr}^{H}(R)$ as an $\mathbf{F}\left[f_{1}, \ldots, f_{n}\right]$-module is generated by $H$-invariants of degree at most

$$
\left(\sum_{i=1}^{n} \operatorname{deg}\left(f_{i}\right)\right)-n
$$

Now for each $Q \leq H$ with $Q \neq 1$ and each $i$ with $1 \leq i \leq n_{Q}$, let

$$
\prod_{\sigma \in G / Q}\left(y-\sigma\left(a_{Q, i}\right)\right)=\sum_{j=0}^{|G: Q|} a_{Q, i, j} y^{j} .
$$

Then for any $j$ with $0 \leq j \leq|G: Q|$,

$$
\operatorname{deg}\left(a_{Q, i, j}\right)=\operatorname{deg}\left(a_{Q, i}\right) \cdot(|G: Q|-j) \leq \beta_{Q} \cdot|G: Q| \leq \bar{\beta}(H) .
$$

Let

$$
A=\mathbf{F}\left[a_{Q, i, j}, f_{s}\left|1<Q \leq H, 1 \leq i \leq n_{Q}, 0 \leq j \leq\right| G: Q \mid, 1 \leq s \leq n\right] .
$$

Then $A$ is a subalgebra of $R^{G}$ and

$$
\beta(A) \leq \max \{\bar{\beta}(H), \mathrm{d}(f)\} .
$$

By Lemma 2.1 we see that $R^{H}$ as an $A$-module is generated by the invariants of degree at most

$$
\left(\sum_{i=1}^{n} \operatorname{deg}\left(f_{i}\right)\right)-n
$$

and the invariants

$$
\operatorname{Tr}_{Q}^{H}\left(a_{Q, 1}^{\alpha_{Q, 1}} \cdots a_{Q, n_{Q}}^{\alpha_{Q, n_{Q}}}\right)
$$

with $1<Q \leq H$ and $0 \leq \alpha_{Q, i} \leq|G: Q|-1$. Now

$$
\begin{aligned}
\operatorname{deg}\left(a_{Q, 1}^{\alpha_{Q, 1}} \cdots a_{Q, n_{Q}}^{\alpha_{Q, n_{Q}}}\right) & \leq n_{Q} \cdot \beta_{Q} \cdot(|G: Q|-1) \\
& \leq e(H) \cdot \beta(H) .
\end{aligned}
$$

It follows that $R^{H}$ as an $A$-module is generated by homogeneous invariants of degree at most

$$
\max \left\{e(H) \cdot \beta(H),\left(\sum_{i=1}^{n} \operatorname{deg}\left(f_{i}\right)\right)-n\right\} .
$$

Now

$$
\operatorname{Tr}_{H}^{G}: R^{H} \longrightarrow R^{G}
$$

is a surjective degree-preserving $A$-homomorphism, so $R^{G}$ as an $A$-module is also generated by homogeneous invariants of degree at most

$$
\max \left\{e(H) \cdot \beta(H),\left(\sum_{i=1}^{n} \operatorname{deg}\left(f_{i}\right)\right)-n\right\} .
$$

Again, since the algebra generators of $A$ together with the $A$-module generators of $R^{G}$ generate $R^{G}$ as an algebra, the result follows. 
Recently, two general degree bounds were obtained, one by Derksen and Kemper and the other by Karagueuzian and Symonds. Derksen and Kemper's result says that for any finite group $G \leq G L(V)$,

$$
\beta\left(R^{G}\right) \leq n(|G|-1)+|G|^{n \cdot 2^{n-1}+1} \cdot n^{2^{n-1}+1} ;
$$

see [5 Theorem 3.9.11]. Karagueuzian and Symonds' result says that if $\mathbf{F}=\mathbf{F}_{q}$ is a finite field, then for any finite group $G \leq G L(V)$,

$$
\beta\left(R^{G}\right) \leq \frac{q^{n}-1}{q-1}(n q-n-1)\left(2 q^{2}-q-2 \text { if } n=2\right) ;
$$

see [12] Theorem 15.4].

Note that the bound (2.1) depends on the order of the group, the dimension of the representation, and not the field. The bound (2.2) depends on the dimension of the representation, the field, and not the group. The bound of Theorem 2.3 depends on more things.

We now give an example to illustrate Theorem 2.3.

For any integer $m>0$, let $m V$ denote the direct sum of $m$ copies of the $\mathbf{F} G$ module $V$. Note that $m V$ is an $\mathbf{F} G$-module by diagonal action. The $\operatorname{ring} \mathbf{F}[m V]^{G}$ is called the ( $m$-dimensional) vector invariant ring of $G$ [17. More discussion of vector invariant rings can be found in [14], [2], 3] and [4].

Example 2.4. Let $\mathbf{F}=\mathbf{F}_{p}, n=2$ and $G=G L(V)$. Thus $|G|=\left(p^{2}-1\right)\left(p^{2}-p\right)$. For $m>0$, let $x_{i}, y_{i}$ be a basis of the $i$-th copy of $m V^{*}, 1 \leq i \leq m$. Thus $\mathbf{F}[m V]=\mathbf{F}\left[x_{i}, y_{i} \mid 1 \leq i \leq m\right]$. Let $\sigma \in G$ be such that $\sigma x_{i}=x_{i}, \sigma y_{i}=y_{i}+x_{i}$, $1 \leq i \leq m$, and let $H=\langle\sigma\rangle$. Then $H$ is a Sylow $p$-subgroup of $G$. By [3, Theorem, p. 4],

$$
\mathbf{F}[m V]^{H}=\mathbf{F}\left[x_{i}, N\left(y_{i}\right), u_{i j}, \operatorname{Tr}^{H}(z) \mid 1 \leq i \leq m, 1 \leq i<j \leq m\right]
$$

where $N\left(y_{i}\right)=y_{i}^{p}-x_{i}^{p-1} y_{i}, u_{i j}=x_{i} y_{j}-x_{j} y_{i}$, and $z$ divides $\left(y_{1} y_{2} \cdots y_{m}\right)^{p-1}$. (2.3) suggests that $\beta\left(\mathbf{F}[m V]^{H}\right)=m(p-1)$. This is indeed the case if $m>2$ (see [14, p. 31]). We have

$$
\mathbf{F}[m V]^{H} / \operatorname{Tr}^{H}(\mathbf{F}[m V])=\mathbf{F}\left[\overline{x_{i}}, \overline{N\left(y_{i}\right)}, \overline{u_{i j}} \mid 1 \leq i \leq m, 1 \leq i<j \leq m\right] .
$$

So,

$$
\begin{gathered}
e(H)=n_{H} \leq 2 m+\left(\begin{array}{c}
m \\
2
\end{array}\right), \\
\beta(H)=\beta_{H} \cdot(|G: H|-1) \leq p \cdot\left((p-1)\left(p^{2}-1\right)-1\right)=|G|-p, \\
\bar{\beta}(H)=\beta_{H} \cdot|G: H| \leq|G|,
\end{gathered}
$$

and

$$
e(H) \cdot \beta(H) \leq \frac{m(m+3)}{2} \cdot(|G|-p) .
$$

Now for each $i$, let $f_{i}=d_{2,0}, g_{i}=d_{2,1} \in \mathbf{F}\left[x_{i}, y_{i}\right]^{G}$ be the Dickson polynomials (see [15, p. 240]). Then $\left\{f_{i}, g_{i} \mid 1 \leq i \leq m\right\}$ is a homogeneous system of parameters of $\mathbf{F}[m V]^{G}$. Furthermore, $\operatorname{deg} f_{i}=p^{2}-1, \operatorname{deg} g_{i}=p^{2}-p$ and

$$
\sum_{i=1}^{m}\left(\operatorname{deg} f_{i}+\operatorname{deg} g_{i}\right)-2 m=m\left(2 p^{2}-p-3\right) .
$$

Now from

$$
\frac{m(m+3)}{2}(|G|-p) \geq 2(|G|-p) \geq|G|
$$


and

$$
\frac{m(m+3)}{2}(|G|-p) \geq 2 m(|G|-p)=2 m p^{2}\left(p^{2}-p-1\right) \geq 2 m p^{2} \geq m\left(2 p^{2}-p-3\right)
$$

we see that

$$
\begin{aligned}
& \max \left\{\frac{m(m+3)}{2}(|G|-p),|G|, p^{2}-1, m\left(2 p^{2}-p-3\right)\right\} \\
= & \frac{m(m+3)}{2}(|G|-p) .
\end{aligned}
$$

So by Theorem 2.3 we have

$$
\beta\left(\mathbf{F}[m V]^{G}\right) \leq \frac{m(m+3)}{2}(|G|-p) .
$$

We note that for the above example the bound of Theorem 2.3 is better than the bound (2.1) and, if $m>2$, better than the bound (2.2). In fact, from $|G|^{n \cdot 2^{n-1}+1} \geq$ $|G|-p$ and $n^{2^{n-1}+1}=(2 m)^{2^{2 m-1}+1} \geq(2 m)^{3} \geq \frac{m(m+3)}{2}$ we see that $(2.4)$ is better than (2.1). To see that (2.4) is better than (2.2) if $m>2$, note that

$$
\begin{aligned}
\frac{p^{n}-1}{p-1}(n p-n-1) & =\frac{p^{2 m}-1}{p-1}(2 m p-2 m-1) \\
& \geq \frac{p^{2 m}-1}{p-1}(m p-m) \\
& =m\left(p^{2 m}-1\right) \\
& \geq m\left(p^{2 m}-p^{2 m-1}-p^{2 m-2}\right) \\
& =m p^{2 m-4}(|G|-p) .
\end{aligned}
$$

Since $m>2$, we have $m p^{2 m-4} \geq m^{2} \geq \frac{m(m+3)}{2}$, as easily seen. So we get

$$
\frac{p^{2 m}-1}{p-1}(2 m p-2 m-1) \geq \frac{m(m+3)}{2}(|G|-p) \text {. }
$$

\section{Applications to $p$-Permutation modules}

Let $p>0$. As an application of the above theorem, we now consider $p$-permutation modules. Recall that the $\mathbf{F} G$-module $V$ is called a $p$-permutation module (or a trivial source module) if for one (hence all) $p$-Sylow subgroups $P$ of $G, V$ is an ordinary permutation module. It is known that $V$ is a $p$-permutation module if and only if it is a direct summand of a permutation module for $G$.

Assume $P \leq G L(V)$ is a non-trivial $p$-Sylow subgroup which permutes the basis $\left\{x_{1}, \ldots, x_{n}\right\}$ of $V^{*}$. Then for any non-trivial subgroup $H \leq P$,

$$
n_{H} \leq n+1-p
$$

since $H$ has at least one orbit in $\left\{x_{1}, \ldots, x_{n}\right\}$ which has length at least $p$. So,

$$
e(P) \leq n+1-p \text {. }
$$

Furthermore,

$$
\beta_{H} \leq|H|
$$

So,

$$
\beta_{H} \cdot(|G: H|-1) \leq|G|-p .
$$


Thus,

$$
\beta(P) \leq|G|-p
$$

and

$$
\bar{\beta}(P) \leq|G| .
$$

From these observations we obtain

Corollary 3.1. Assume that $V$ is a p-permutation module for $G$, and $\left\{f_{1}, \ldots, f_{n}\right\}$ is a homogeneous system of parameters for $R^{G}$. Then

$$
\beta\left(R^{G}\right) \leq \max \left\{(n+1-p)(|G|-p),|G|, d(f),\left(\sum_{i=1}^{n} \operatorname{deg}\left(f_{i}\right)\right)-n\right\} .
$$

In particular,

$$
\beta\left(R^{G}\right) \leq \max \{|G|, n(|G|-1)\} .
$$

Proof. The first part has been shown. For the second part, note that $\beta\left(R^{G}\right)$ does not change if we extend the ground field $\mathbf{F}$ (see [2, Lemma 2.4]). So we may assume that $\mathbf{F}$ is algebraically closed. Thus by Dade's algorithm (see [16], or [5. Proposition 3.3.2]) we can find a homogeneous system of parameters of degrees $\leq|G|$, hence the result.

Remark 3.2. The second inequality in the above corollary was also proved by Fleischmann (see 7, Proposition 2.1.2]), and it has been conjectured to be true for any group (see [5, Remark 3.7.12]). Hughes and Kemper (see 11, Theorem 2.17]) proved this conjecture for the case where $|G|>1$ and $p^{2}$ does not divide $|G|$, and Broer (see [1], or [5, Corollary 3.9.9]) proved this conjecture for the case where $R^{G}$ is Cohen-Macaulay.

The following example shows that the first bound can be better than the second bound in the above corollary.

Example 3.3. Let $V^{*}=\operatorname{span}\left\{x_{1}, \ldots, x_{5}\right\}$ be a five-dimensional vector space over $\mathbf{F}$, a field of characteristic 3, and let $G=\langle(12),(345)\rangle\left\langle S_{5}\right.$ act as a permutation group on $\left\{x_{1}, \ldots, x_{5}\right\}$. We have $|G|=6$. Let $f_{1}=x_{1}+x_{2}, f_{2}=x_{1} x_{2}, f_{3}=x_{3}+$ $x_{4}+x_{5}, f_{4}=x_{3} x_{4}+x_{3} x_{5}+x_{4} x_{5}$, and $f_{5}=x_{3} x_{4} x_{5}$. Then $\left\{f_{1}, \ldots, f_{5}\right\}$ is a homogeneous system of parameters for $R^{G}$. We see that

$$
\max \left\{(n+1-p)(|G|-p),|G|, \mathrm{d}(f),\left(\sum_{i=1}^{n} \operatorname{deg}\left(f_{i}\right)\right)-n\right\}=9 .
$$

However, $\max \{|G|, n(|G|-1)\}=25$.

We remark that if $p=2$ in Example 2.4, then $H$ acts on the basis $\left\{x_{i}+y_{i}, y_{i} \mid\right.$ $1 \leq i \leq m\}$ of $m V^{*}$, as well as on the dual basis to $\left\{x_{i}+y_{i}, y_{i} \mid 1 \leq i \leq m\right\}$, as a permutation group. So $m V$ is a 2-permutation module for $G$. Thus by Corollary 3.1 , when $m>1$ we have

$$
\beta\left(\mathbf{F}[m V]^{G}\right) \leq 4(2 m-1) .
$$

(If $m=1,(3.3)$ is still true because $\mathbf{F}[V]^{G}$ is the polynomial ring generated by the Dickson polynomials (see [15, Theorem 8.1.6]) and thus $\beta\left(\mathbf{F}[V]^{G}\right)=3$.) 
Goebel (see [10, Theorem 3.11]) showed that for any permutation group $G \subseteq S_{n}$ and any commutative ring $A$,

$$
\beta\left(A\left[X_{1}, \ldots, X_{n}\right]^{G}\right) \leq \frac{n(n-1)}{2} .
$$

This result implies that if $G$ is any finite group and $V$ is the regular module for $G$, then

$$
\beta\left(R^{G}\right) \leq \max \left\{|G|, \frac{|G|}{2}(|G|-1)\right\} .
$$

We can recover this result by using Theorem 2.3.

Corollary 3.4. Let $G$ be any finite group and $V$ be the regular module for $G$. Then

$$
\beta\left(R^{G}\right) \leq \max \left\{|G|, \frac{|G|}{2}(|G|-1)\right\} .
$$

Proof. Let $S=\left\{x_{g} \mid g \in G\right\}$ be the basis of $V^{*}$ and let $e_{1}, \ldots, e_{n}$ be the elementary symmetric polynomials in the $x_{g}$ 's. Then $\left\{e_{1}, \ldots, e_{n}\right\}$ is a homogeneous system of parameters for $R^{G}$. Let $P$ be a $p$-Sylow subgroup of $G$. If $P=1$, then $R^{G}$ is Cohen-Macaulay and thus by [5] Theorem 3.9.8],

$$
\begin{aligned}
\beta\left(R^{G}\right) & \leq \max \left\{\max \left\{e_{1}, \ldots, e_{n}\right\},\left(\sum_{i=1}^{n} \operatorname{deg}\left(e_{i}\right)\right)-|G|\right\} \\
& =\max \left\{|G|, \frac{|G|}{2}(|G|-1)\right\} .
\end{aligned}
$$

So we assume $|P|>1$. Since $V$ is regular, for any subgroup $H \leq P$ and $g \in G$ we have $\left|H x_{g}\right|=|H|$. So

and thus

$$
n_{H} \leq|G: H|,
$$

$$
e(P) \leq \frac{|G|}{p} .
$$

By (3.1) we have

$$
e(P) \cdot \beta(P) \leq \frac{|G|}{p}(|G|-p) \leq \frac{|G|}{2}(|G|-1) .
$$

From (3.2) and Theorem 2.3 the result follows.

\section{ACKNOWLEDGEMENTS}

The author is grateful to Eddy Campbell, Ian Hughes and David Wehlau for their help and support, and to Gregor Kemper for suggesting some changes. He is also grateful to the referee for helpful comments.

\section{REFERENCES}

[1] A. Broer, Remarks on invariant theory of finite groups, Preprint, Université de Montréal, Montréal, (1997).

[2] H. E. A. Campbell, A. V. Geramita, I. P. Hughes, R. J. Shank and D. L. Wehlau, NonCohen-Macaulay vector invariants and a Noether bound for a Gorenstein ring of invariants, Canad. Math. Bull. 42(2)(1999), 155-161. MR1692004 (2000b:13005)

[3] H. E. A. Campbell and I. P. Hughes, Vector invariants of $U_{2}\left(\mathbf{F}_{p}\right):$ A proof of a conjecture of Richman, Adv. in Math. 126(1997), 1-20. MR1440251 (98c:13007)

[4] Jianjun Chuai, Two-dimensional vector invariant rings of Abelian p-groups, J. of Alg. 266(1)(2003), 362-373. MR.1994545 (2004e:13010) 
[5] H. Derksen and G. Kemper, Computational invariant theory, Springer, (2002). MR1918599 (2003g:13004)

[6] J. A. Eagon and M. Hochster, Cohen-Macaulay rings, invariant theory, and the generic perfection of determinantal loci, Amer. J. Math. 93(1971), 1020-1058. MR0302643 (46:1787)

[7] P. Fleischmann, On invariants theory of finite groups, Preprint, Invariant Theory Workshop at Queen's University, (2002).

[8] P. Fleischmann, The Noether bound in invariant theory of finite groups, Adv. in Math. 156(2000), 23-32. MR1800251 (2001k:13009)

[9] J. Fogarty, On Noether's bound for polynomial invariants of a finite group, Elec. Res. Announ. of the Amer. Math. Soc. 7(2001), 5-7. MR1826990 (2002a:13002)

[10] M. Goebel, Computing bases for rings of permutation-invariant polynomials, J. Symb. Comp., 19 (1995), 285-291. MR1339909 (96f:13006)

[11] I. Hughes and G. Kemper, Symmetric powers of modular representations, Hilbert series and degree bounds, Comm. Alg., 28(4) (2000), 2059-2088. MR.1747371 (2001b:13009)

[12] D. B. Karagueuzian and Peter Symonds, The module structure of a group action on a polynomial ring: the general case, Preprint, (2001).

[13] E. Noether, Der Endlichkeitssatz der Invarianten endlicher Gruppen, Math. Ann. 77(1916), 89-92.

[14] D. R. Richman, On vector invariants over finite fields, Adv. in Math. 81(1990), 30-65. MR,1051222 (91g:15020)

[15] L. Smith, Polynomial invariants of finite groups, A. K. Peter, Ltd. 1995. MR1328644 (96f:13008)

[16] R. P. Stanley, Polynomial invariants of finite groups and their applications to combinatorics, Bull. Amer. Math. Soc. 1(3) (1979), 475-511. MR0526968 (81a:20015)

[17] H. Weyl, The classical groups, Princeton University Press, Princeton, NJ, (1939). MR0000255 $(1: 42 \mathrm{c})$

Department of Mathematics and Statistics, Queen's University, Kingston, Ontario, CANADA K7L 3N6

E-mail address: chuai@mast.queensu.ca 